\title{
LDLR C1725T Gene Polymorphism Frequency in Type 2 Diabetes Mellitus Patients With Dyslipidemia
}

\author{
Zuhal Eroglua, e, Ece Harman ${ }^{\mathrm{b}}$, Egemen Vardarlic, Meral Kayikcioglu ${ }^{\mathrm{d}}$, \\ Asli Tetik Vardarli ${ }^{\mathrm{a}}$
}

\begin{abstract}
Background: Dyslipidemia has a substantial role in the development of cardiovascular diseases in patients with type 2 diabetes mellitus (T2DM). Determining the genetic profile of T2DM patients with dyslipidemia is important in order to reduce the risk of microvascular and macrovascular complications. Low-density lipoprotein receptor (LDLR) plays a critical role in plasma lipoprotein hemostasis. LDLR mutations/polymorphisms cause changes at the lipoprotein level. The objective of this study is to determine the frequency of LDLR (rs179989) polymorphisms in Turkish T2DM patients with dyslipidemia.
\end{abstract}

Methods: The study group consisted of 217 T2DM patients with dyslipidemia including 28 cases with myocardial infarction and 212 healthy controls. Genomic DNA was isolated from venous blood samples and genotype analysis was carried out on the LightCycler ${ }^{\circledR} 480$ instrument. The $\chi^{2}$ test was used to compare genotype distributions.

Results: There were no significant differences in the frequency or allelic distribution of the LDLR C1725T (rs1799898) genotype between the type 2 diabetic dyslipidemia patients and the control group $(\mathrm{P}>0.05)$.

Conclusion: LDLR C1725T polymorphism was not associated with lipid parameters, and dyslipidemia in T2DM patients.

Keywords: Diabetes mellitus; Dyslipidemia; Lipid metabolism; LDLR; C1725T

Manuscript accepted for publication September 13, 2016

aDepartment of Medical Biology, Medicine Faculty, Ege University, Izmir, Turkey

${ }^{b}$ Division of Endocrinology and Metabolism Disease, Department of Internal Medicine, Medical Faculty, Katip Celebi University, Izmir, Turkey

'Department of Neurology, Tinaztepe Hospital, Izmir, Turkey

dDepartment of Cardiology, Faculty of Medicine, Ege University, Izmir, Turkey

${ }^{e}$ Corresponding Author: Zuhal Eroglu, Department of Medical Biology, Ege University Medical School, 35100 Bornova, Izmir, Turkey.

Email: zuhal.eroglu@ege.edu.tr

doi: http://dx.doi.org/10.14740/jocmr2739w

\section{Introduction}

Diabetes mellitus (DM) occurs due to impairments in insulin secretion and/or function and is characterized by increased blood glucose levels (hyperglycemia) [1]. The prevalence of $\mathrm{DM}$ is steadily rising, leading to subsequent increases in many diabetes-related comorbidities [2]. Cardiovascular diseases directly comprise $80 \%$ of the increasing mortality in diabetic individuals, and it has been reported that type 2 diabetic individuals have a 2 - 4 times higher risk of developing cardiovascular disease compared to healthy individuals [3-6]. Due to the high morbidity and mortality in these patients, the prevention of cardiovascular complications has become a primary objective in the management of diabetes [7]. In addition to insulin resistance, other cardiovascular risk factors include dyslipidemia, hypertension, susceptibility to coagulation, obesity, hyperinsulinemia and inflammation $[8,9]$. Dyslipidemia, one of the most important risk factors, includes various pathologic conditions involving abnormal concentration, composition and distribution of lipids. Recent studies have shown that proteins rich in triglycerides such as chylomicrons and very low-density lipoproteins (VLDL) are significant risk factors for coronary artery disease (CAD), demonstrating that dyslipidemia is an important factor in the pathogenesis of cardiovascular disease in patients with DM. The most common phenotypic characteristics of dyslipidemia in type 2 diabetes are high plasma triglyceride concentration, low high-density lipoprotein cholesterol (HDLC) concentration and elevated low-density lipoprotein cholesterol (LDL-C) [8].

Low-density lipoprotein receptors (LDLRs) are cell-surface receptors that regulate cellular LDL-C particle intake by receptor-mediated endocytosis and play an important role in plasma lipoprotein hemostasis [10]. LDLR proteins are coded by the LDLR gene consisting of 18 exons of $45 \mathrm{~kb}$ in length located on chromosome 19p13. Studies performed to date have identified more than 800 large deletions, small deletions, insertions, point mutations, splice site mutations and polymorphisms of LDLR, and it has been determined that these genetic variations alter individuals' lipoprotein levels [11]. It has also been shown that some of these genetic changes increase the risk of dyslipidemia and cardiovascular disease by increasing levels of total cholesterol and LDL-C in some individuals, while other genetic alterations seem to have a protective effect against cardiovascular disease $[12,13]$. 
Table 1. Characteristics and the Biochemical Parameters of Diabetic Dyslipidemia Patients and Control Group

\begin{tabular}{|c|c|c|c|}
\hline & Diabetic dyslipidemia $($ mean \pm SD) $(n=217)$ & Control $($ mean \pm SD) $(n=212)$ & P-value \\
\hline Gender (female/male) & $133 / 84$ & $122 / 90$ & NS \\
\hline Age (years) & $53.2 \pm 9.8$ & $52.8 \pm 9.2$ & NS \\
\hline Fasting glucose $(\mathrm{mg} / \mathrm{dL})$ & $161.9 \pm 72.5$ & $87.7 \pm 11.6$ & $<0.001$ \\
\hline Total cholesterol (mg/dL) & $219.3 \pm 61.8$ & $178.2 \pm 20.7$ & $<0.001$ \\
\hline Triglycerides (mg/dL) & $231.6 \pm 255.8$ & $109.5 \pm 25.9$ & $<0.001$ \\
\hline HDL cholesterol (mg/dL) & $48.5 \pm 13.6$ & $63 \pm 8.9$ & $<0.001$ \\
\hline LDL cholesterol (mg/dL) & $131.6 \pm 42.3$ & $117 \pm 17.9$ & \\
\hline Lipid-lowering therapy (\%) & 68 & - & \\
\hline Oral antidiabetic therapy $(\%)$ & 20 & - & \\
\hline Insulin therapy $(\%)$ & 29 & - & \\
\hline
\end{tabular}

HDL: high-density lipoproteins; LDL: low-density lipoproteins.

In this study, we aimed to investigate the frequency of the LDLR (rs1799898) polymorphism in type 2 diabetes mellitus (T2DM) patients with dyslipidemia and evaluate its association with dyslipidemia.

\section{Materials and Methods}

The study group included a total of 217 patients (133 females and 84 males) diagnosed with type 2 diabetic dyslipidemia in the Endocrinology Outpatient Clinic of Ege University Faculty of Medicine, Department of Internal Diseases between January 2009 and September 2011. The control group consisted of 212 healthy individuals (122 females and 90 males) age- and gender-matched to the study group. Type 2 diabetes diagnosis was based on a fasting blood glucose (FBG) level $>126$ $\mathrm{mg} / \mathrm{dL}$ and/or postprandial glucose level $>200 \mathrm{mg} / \mathrm{dL}$ and HbA1c level $>6.5 \%$. Subjects with triglyceride levels $>200$ $\mathrm{mg} / \mathrm{dL}$ and/or HDL-C levels $<45 \mathrm{mg} / \mathrm{dL}$ were diagnosed with diabetic dyslipidemia. Subjects in the control group did not meet any of these criteria. The study protocol was approved by the Research Ethics Committee of Ege University Faculty of Medicine. Each patient provided a detailed medical history. Biochemical analyses, such as $\mathrm{HbA1c}$, total cholesterol, triglyceride, HDL-C, LDL-C and FBG, were performed with an Olympus AU 2700 chemistry analyzer (Toshiba, Tokyo, Japan).

Blood specimens were collected from each subject into tubes containing ethylenediaminetetraacetic acid (EDTA). Genomic DNA was isolated from peripheral leukocytes of the subjects using MagNA Pure LC DNA Isolation Kit I by MagNA Pure LC DNA isolation instrument (Roche Applied Science). LDLR LightMix ${ }^{\circledR}$ Kit (TIB MOLBIOL) was used to analyze the LDLR C1725T polymorphism. PCR master mix and conditions for LDLR detection were as follows: 10.4 $\mu \mathrm{L} \mathrm{H}_{2} \mathrm{O}, 1.6 \mu \mathrm{L} 25 \mathrm{mM} \mathrm{MgCl}{ }_{2}, 2 \mu \mathrm{L}$ reagent mix (parameterspecific reagents containing primers and simple probe) (TIB MOLBIOL), $1 \mu \mathrm{L}$ LightCycler FastStart DNA Master Hybridization Probes (Roche Applied Sciences), $5 \mu \mathrm{L}$ genomic DNA; denaturation $\left(95^{\circ} \mathrm{C}\right.$ for $\left.10 \mathrm{~s}\right), 45$ cycles amplification $\left(95^{\circ} \mathrm{C}\right.$ for $10 \mathrm{~s} ; 60^{\circ} \mathrm{C}$ for $10 \mathrm{~s} ; 72^{\circ} \mathrm{C}$ for $\left.15 \mathrm{~s}\right)$, melting analysis $(95$ ${ }^{\circ} \mathrm{C}$ for $30 \mathrm{~s}, 40{ }^{\circ} \mathrm{C}$ for $2 \mathrm{~min}$, and $75^{\circ} \mathrm{C} 0 \mathrm{~s}$ ) and cooling step at $40{ }^{\circ} \mathrm{C}$ for $30 \mathrm{~s}$. All experiments were carried out on the LightCycler $^{\circledR} 480$ Instrument (Roche Applied Science, Mannheim, Germany). Polymorphic alleles were identified by the specific melting temperatures of the resulting amplicons, as individuals with two copies of the $\mathrm{C}$ allele (CC) showed a single melting peak at $64{ }^{\circ} \mathrm{C}$, individuals with two copies of the T allele (TT) showed a single melting peak at $57{ }^{\circ} \mathrm{C}$, and individuals with both alleles (CT) showed two melting peaks at 57 and $64{ }^{\circ} \mathrm{C}$ in the melting curve analysis.

The biochemical data of type 2 diabetic dyslipidemia patients and healthy control group were compared in terms of the genotype distribution of the LDLR C1725T polymorphism.

\section{Statistical analysis}

All statistical analyses were performed using SPSS for Windows (Version 18.0, SPSS, Chicago, IL, USA). The data were presented as percentages for discrete variables and as mean \pm standard deviation (SD) for continuous variables. A P value of $<0.05$ (two-sided) was considered statistically significant. Comparisons between groups were made by $t$-test, and discrete variables were compared by Chi-square analysis.

\section{Results}

The study population comprised 217 unrelated Turkish type 2 diabetic dyslipidemia cases (133 females and 84 males), including 28 subjects (13 female, 15 males) with myocardial infarction (MI) and 212 controls (122 females and 90 males). The comparison of clinical characteristics and biochemical parameters of the groups is shown in Table 1. FBG, total cholesterol, triglycerides, and LDL-C levels were significantly higher in the diabetic dyslipidemia group than in the control group; whereas HDL-C levels were significantly lower $(\mathrm{P}<0.001)$. Patients in the diabetic dyslipidemia group were treated with oral antidiabetics $(n=68)$, insulin $(n=20)$ and lipid-lowering 
Table 2. Genotype Distribution and Allele Frequency of LDLR C1725T (rs1799898) Gene Polymorphisms in Diabetic Dyslipidemia Patients and the Control Group

\begin{tabular}{lllll}
\hline Gene/SNP & Genotypes haplotype & Patients, n (\%) & Control, n (\%) & P-value \\
\hline LDLR C1725T (rs1799898) & CC & $143(66.2)$ & $142(67.0)$ & 0.256 \\
& CT & $70(32.4)$ & $62(29.2)$ & \\
& TT & $3(1.4)$ & $8(3.8)$ \\
C & 356 & 346 & 78 \\
\hline
\end{tabular}

agents $(\mathrm{n}=29)$ (Table 1).

Wild-type (CC), heterozygous (CT) and polymorphic (TT) genotype distributions of the LDLR (rs1799898) polymorphism were found to be $66.2 \%, 32.4 \%$, and $1.4 \%$ in the type 2 diabetic dyslipidemia group and $67 \%, 29.2 \%$, and $3.8 \%$ in the control group, respectively. There was no significant difference between the groups' distributions of LDLR (rs1799898) polymorphisms (Table 2).

Moreover, we also compared genotype distribution of LDLR (rs1799898) gene polymorphism with type 2 diabetic dyslipidemia patients and type 2 diabetic dyslipidemia patients with MI. For the LDLR C1725T polymorphism, distribution of the $\mathrm{CC}, \mathrm{CT}$, and TT genotypes was found to be $66 \%, 32.4 \%$, and $1.6 \%$ and $67.9 \%, 32.1 \%$, and $0 \%$ in the type 2 diabetic dyslipidemia patients and type 2 diabetic dyslipidemia patients with MI groups, respectively (Table 3). However, we did not find significant association for frequencies of $\mathrm{C} 1725 \mathrm{~T}$ genotypes.

A comparison of biochemical parameters such as $\mathrm{HbAlc}$, FBG, total cholesterol, triglycerides, HDL-C, and LDL-C levels between diabetic dyslipidemia patients and control group according to LDLR (rs1799898) genotype revealed no significant correlations between lipid parameters, plasma glucose, $\mathrm{HbA1c}$ levels and genotype distribution $(\mathrm{P}>0.005)$.

\section{Discussion}

Modern changes in lifestyle and nutrition habits are increasing the incidence of T2DM considerably, together with the influence of hereditary and environmental factors. The relation between DM and serum lipid profiles has been discussed for the last decade [14]. From studies of T2DM patients, it is known that diabetic dyslipidemia occurs not only as a disruption of lipoprotein metabolism resulting from changes in the quantitative and qualitative characteristics of lipoproteins, but may also develop due to genetic and environmental factors. Therefore, determining the genetic profile of diabetic patients with dyslipidemia is considered important in terms of reducing the risk of possible microvascular and macrovascular complications. To date, no previous studies have reported the effects of LDLR (rs1799898) polymorphism in diabetic dyslipidemia patients. This study is the first comprehensive study investigating the frequency of LDLR (rs1799898) polymorphisms in Turkish type 2 diabetic dyslipidemia patients. According to our analysis, the wild-type, heterozygote, and polymorphic genotype rates are $66.2 \%, 32.4 \%$, and $1.4 \%$ in type 2 diabetic dyslipidemia patients and $67 \%, 29.2 \%$, and $3.8 \%$ in controls, respectively.

LDLR is a membrane glycoprotein which regulates the uptake and degradation of cholesterol-rich lipoproteins by receptor-mediated endocytosis [10, 12]. LDLR mutations/polymorphisms change individuals' LDLR protein structures and functions and lead to the formation of different clinical phenotypes $[11,15,16]$. Several studies have investigated whether common SNPs in LDLR such as rs12983082, rs2738446, rs1799898, rs9789302, rs5925, and rs688 contribute to individual variations in serum lipid concentrations. Tejedor et al investigated the effects of LDLR (rs1799898) polymorphisms in familial hypercholesterolemia patients and did not detect any remarkable phenotypic effects on disease development, as in our study [17]. Jamaldini et al investigated the effects of LDLR C1725T polymorphisms on the development of CAD and reported that CC, CT, and TT genotype rates were $79.41 \%$, $17.65 \%$, and $2.94 \%$ in CAD patients and $64.08 \%, 32.04 \%$, and $3.88 \%$ in the control group, respectively. In contrast to the current study, Jamaldini supported that the CT or TT genotypes of C1725T (rs 1799898) SNP may have a protective effect against CAD [13]. In our study, only 28 type 2 diabetic dyslipidemia cases had MI. Therefore, the number of the patients with MI is too small to determine the association between LDLR C1725T gene polymorphism and cardiovascular disease in our study.

In genetically heterogeneous populations, obtaining contradictory results can be attributed to regulation by gene-gene and gene-environment interactions and/or diversity of patients' characteristics. Type 2 diabetic dyslipidemia is a complex dis-

Table 3. Genotype Distribution of LDLR C1725T (rs1799898) Gene Polymorphisms in Type 2 Diabetic Dyslipidemia Patients and Type 2 Diabetic Dyslipidemia Patients With MI

\begin{tabular}{lllll}
\hline Gene/SNP & Genotypes haplotype & Patients, n (\%) & Patients with MI, n (\%) & P-value \\
\hline LDLR C1725T (rs1799898) & CC & $124(66.0)$ & $19(67.9)$ & $>0.05$ \\
& CT & $61(32.4)$ & $9(32.1)$ & \\
& TT & $3(1.6)$ & $0(0)$ & \\
\hline
\end{tabular}


ease influenced by genetic and environmental factors. Therefore, it is difficult to definitively identify pathogenetic factors. Further studies with larger patient numbers in different/same populations are needed. In future studies, other LDLR polymorphisms/mutations should be investigated in order to determine their effects on individual variations in lipoprotein levels and disease development.

\section{Financial Disclosure}

No competing financial interests exist.

\section{Conflicts of Interest}

There are no conflicts of interest.

\section{Author Contributions}

Concept: ZE, EH, and ATV. Design: ATV and ZE. Supervision: ZE and ATV. Materials: EH and EV. Data collection and/ or processing: ATV, EH, EV, and MK. Analysis and/or interpretation: ATV and MK. Literature search: ZE and ATV. Writing: ZE and ATV. Critical review: AZ, ZE, VBC, EV, and ASK.

\section{References}

1. Diagnosis and classification of diabetes mellitus. Diabetes Care. 2011;34(Suppl 1):S62-69.

2. Wild S, Roglic G, Green A, Sicree R, King H. Global prevalence of diabetes: estimates for the year 2000 and projections for 2030. Diabetes Care. 2004;27(5):10471053.

3. Duby JJ, Campbell RK, Setter SM, White JR, Rasmussen KA. Diabetic neuropathy: an intensive review. Am J Health Syst Pharm. 2004;61(2):160-173; quiz 175-166.

4. Goldberg RB. Cardiovascular disease in patients who have diabetes. Cardiol Clin. 2003;21(3):399-413, vii.

5. Kikkawa R, Koya D, Haneda M. Progression of diabetic nephropathy. Am J Kidney Dis. 2003;41:19-21.

6. Winer N, Sowers JR. Epidemiology of diabetes. J Clin Pharmacol. 2004;44(4):397-405.
7. Amos AF, McCarty DJ, Zimmet P. The rising global burden of diabetes and its complications: estimates and projections to the year 2010. Diabet Med. 1997;14(Suppl 5):S1-85.

8. Mooradian AD. Dyslipidemia in type 2 diabetes mellitus. Nat Clin Pract Endocrinol Metab. 2009;5(3):150-159.

9. Ven Murthy MR, Julien P, Singh P, Levy E. Human lipoprotein lipase deficiency: does chronic dyslipidemia lead to increased oxidative stress and mitochondrial DNA damage in blood cells? Acta Biochim Pol. 1996;43(1):227240.

10. Muallem H, North KE, Kakoki M, Wojczynski MK, Li X, Grove M, Boerwinkle E, et al. Quantitative effects of common genetic variations in the 3'UTR of the human LDL-receptor gene and their associations with plasma lipid levels in the Atherosclerosis Risk in Communities study. Hum Genet. 2007;121(3-4):421-431.

11. Wang B, Zhao H, Zhou L, Dai X, Wang D, Cao J, Niu W. Association of genetic variation in apolipoprotein $\mathrm{E}$ and low density lipoprotein receptor with ischemic stroke in Northern Han Chinese. J Neurol Sci. 2009;276(1-2):118122.

12. Boes E, Coassin S, Kollerits B, Heid IM, Kronenberg F. Genetic-epidemiological evidence on genes associated with HDL cholesterol levels: a systematic in-depth review. Exp Gerontol. 2009;44(3):136-160.

13. Jamaldini SH, Babanejad M, Mozaffari R, Nikzat N, Jalalvand K, Badiei A, Sanati H, et al. Association of polymorphisms at LDLR locus with coronary artery disease independently from lipid profile. Acta Med Iran. 2014;52(5):352-359.

14. Dixit AK, Dey R, Suresh A, Chaudhuri S, Panda AK, Mitra A, Hazra J. The prevalence of dyslipidemia in patients with diabetes mellitus of ayurveda Hospital. J Diabetes Metab Disord. 2014;13:58.

15. Silva S, Alves AC, Patel D, Malho R, Soutar AK, Bourbon $M$. In vitro functional characterization of missense mutations in the LDLR gene. Atherosclerosis. 2012;225(1):128-134.

16. Miller M, Zhan M. Genetic determinants of low highdensity lipoprotein cholesterol. Curr Opin Cardiol. 2004;19(4):380-384.

17. Tejedor MT, Cenarro A, Tejedor D, Stef M, Palacios L, de Castro I, Garcia-Otin AL, et al. New contributions to the study of common double mutants in the human LDL receptor gene. Naturwissenschaften. 2011;98(11):943-949. 